\title{
A rare case of hemobilia associated with aneurysms of the celiac trunk, the hepatic artery, and the splenic artery
}

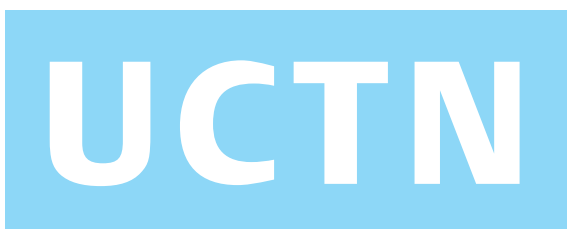

A 49-year-old woman presented with acute gastrointestinal hemorrhage. She had a history of a melena with negative gastroscopy and colonoscopy examinations. On admission, her hemoglobin level was $9.4 \mathrm{~g} / \mathrm{dl}$, and the indices of cholestasis and hepatic cytolysis were normal. Urgent gastroscopy revealed fresh blood in the duodenum without a clear source of bleeding, but subsequent duodenoscopy showed evidence of active bleeding from the papilla of Vater (Figure $\mathbf{1}$ ). Abdominal computed tomography revealed a large thrombosed aneurysm of the splenic artery associated with aneurysms of the celiac trunk and of the common hepatic artery (Figure $\mathbf{2}$ ). The melena persisted and so angiography was performed, which confirmed the presence of the three aneurysms (Figure 3). Embolization was performed by the insertion of 38 metallic coils in the splenic artery, the gastric artery, and the celiac trunk. The aneurysms thrombosed completely, as shown in the angiographic control 2 weeks later (Figure 4). The patient recovered well and was discharged 1 week later. No further bleeding was observed over 5 months of follow-up.

Hemobilia usually presents with melena (90\%), hematemesis (72\%), upper abdominal pain (71\%), or obstructive jaundice (60\%); the classic triad of bleeding, pain, and obstructive jaundice occurs in about $22 \%$ of cases [1]. Our patient presented mainly with melena. In around $10 \%$ of patients with hemobilia, the condition is secondary to ruptured hepatic artery aneurysms [2]. Splenic artery aneurysm is the most common visceral artery aneurysm (60\%); the incidence of hepatic artery aneurysm is $20 \%$ and the incidence of celiac artery aneurysm is $4 \%$ [3].

Upper gastrointestinal endoscopy is often negative in cases of hemobilia because of the intermittent nature of the bleeding. However, duodenoscopy reveals positive findings in more than $50 \%$ of cases [4],

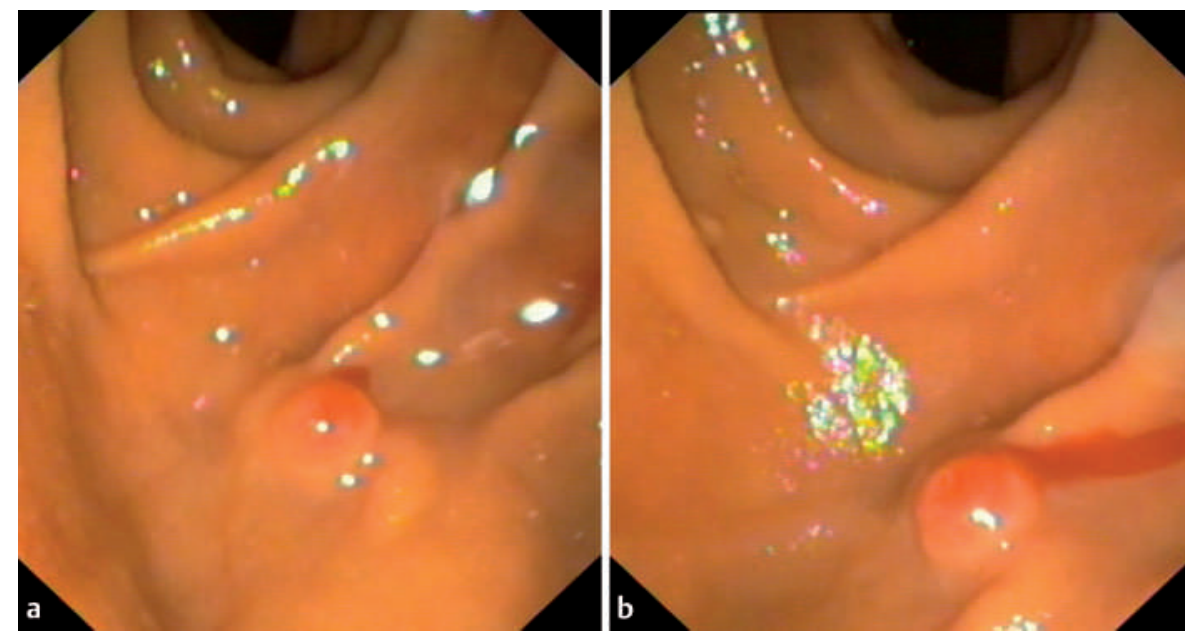

Figure 1 Duodenoscopy was performed with a lateral-viewing endoscope and showed evidence of active bleeding from the papilla of $\operatorname{Vater}(\mathbf{a}, \mathbf{b})$.
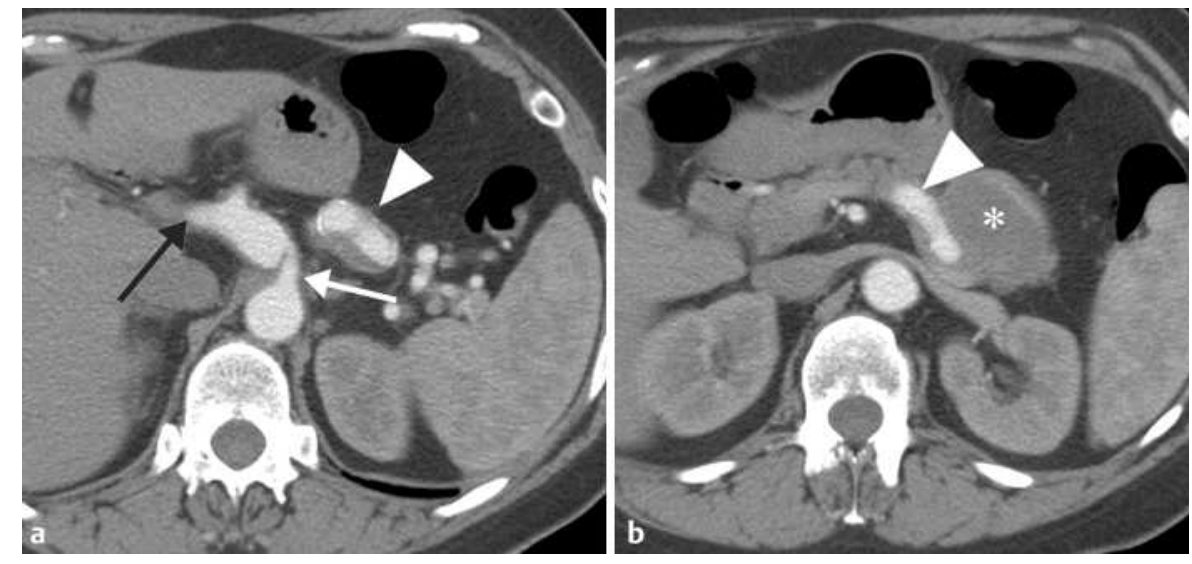

Figure 2 Abdominal computed tomographic scans after injection of a contrast agent. a This view shows an aneurysm of the celiac trunk with a neck about $2 \mathrm{~cm}$ in length (white arrow); an aneurysm of the common hepatic artery, which was obstructed at its origin (black arrow); and an aneurysmal sac located

and selective superior mesenteric angiography can both localize the origin of the bleeding and provide an opportunity for arterial embolization with few complications [5]. in the splenic arterial tract (white arrowhead). $\mathbf{b}$ This view shows the splenic artery aneurysm (white arrowhead), which was dilated to a maximum diameter of $50 \mathrm{~mm}$ because of a large intraluminal thrombus (asterisk).

Endoscopy_UCTN_Code_CCL_1AZ_2AM

\section{G. Traversa', M. Zippi' ${ }^{1}$, A. Bruni ${ }^{2}$,}

M. Mancuso² ${ }^{2}$ P. Di Stefano ${ }^{3}$,

G. Occhigrossi ${ }^{1}$

${ }^{1}$ Department of Digestive Endoscopy and Gastroenterology, Sandro Pertini Hospital, Rome, Italy

2 Department of Interventional Radiology, Sandro Pertini Hospital, Rome, Italy 


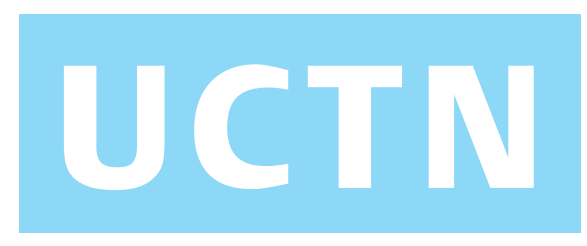

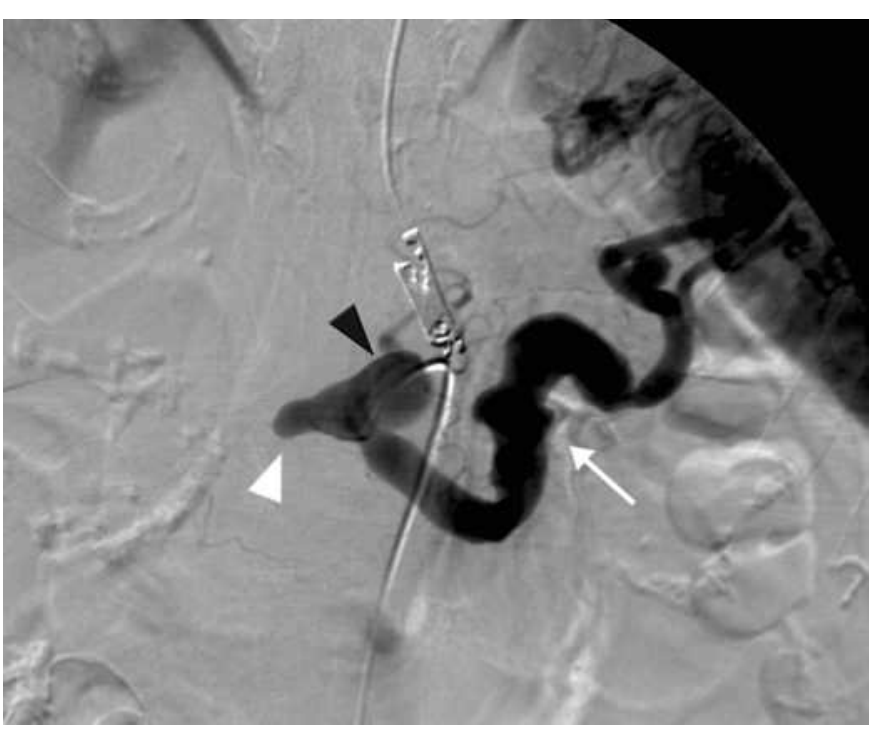

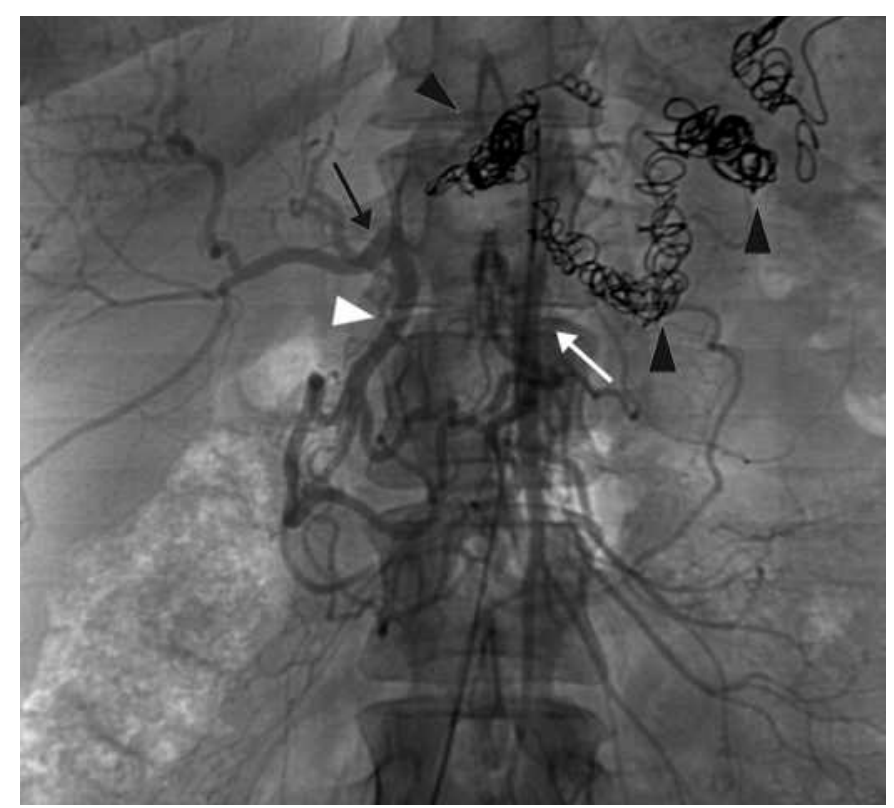

Figure 3 Selective superior mesenteric artery angiography showed the obstruction of the common hepatic artery (white arrowhead), the aneurysmal dilatation of the celiac trunk (black arrowhead), and parietal alterations of the splenic artery (white arrow) caused by the presence of the aneurysm.

Figure 4 Post-embolization selective superior mesenteric artery angiography demonstrated the revascularization of the hepatic artery proper (black arrow) through the gastroduodenal artery (white arrowhead), with inverted flow coming off the superior mesenteric artery (white arrow). The image also shows the metallic coils in the celiac and splenic arteries (black arrowheads).
${ }^{3}$ Department of Surgery, Sandro Pertini Hospital, Rome, Italy.

\section{References}

${ }^{1}$ Forlee MV, Krige JE, Welman CJ, Beningfield SJ. Haemobilia after penetrating and blunt liver injury: treatment with selective hepatic artery embolization. Injury 2004; 35 : $23-28$

2 Liu TT, Hou MC, Lin HC et al. Life-threatening hemobilia caused by hepatic artery pseudoaneurysm: a rare complication of chronic cholangitis. World J Gastroenterol 2003; 9: $2883-2884$

${ }^{3}$ Janzen RM, Simpson WT. Visceral artery aneurysm. Can J Surg 2000; 43: 301 - 302

${ }^{4}$ Curet P, Baumer R, Roche A et al. Hepatic hemobilia of traumatic or iatrogenic origin: recent advances in diagnosis and therapy, review of the literature from 1976 to 1981. World J Surg 1984; 8: 2 -8

${ }^{5}$ Madoff DC, Denys A, Wallace MJ et al. Splenic arterial interventions: anatomy, indications, technical considerations, and potential complications. Radiographics 2005; 25: S191 - S211

\section{Corresponding Author}

\section{G. Occhigrossi, M.D.}

Department of Digestive Endoscopy and Gastroenterology

Sandro Pertini Hospital

Via dei Monti Tiburtini, 385

\section{Rome}

Italy

Fax: +39-06-41735005

E-mail: giuseppe.occhigrossi@ aslromab.it 